\title{
Ball Screw Fault Detection and Location Based on Outlier and Instantaneous Rotational Frequency Estimation
}

\author{
Liang Guo $\mathbb{D}^{1},{ }^{1,2}$ Yingqi Huang, ${ }^{3}$ Hongli Gao, ${ }^{1,2}$ and Li Zhang $\mathbb{D}^{1,2}$ \\ ${ }^{1}$ Engineering Research Center of Advanced Driving Energy-saving Technology, Ministry of Education, \\ Southwest Jiaotong University, Chengdu 610031, China \\ ${ }^{2}$ School of Mechanical Engineering, Southwest Jiaotong University, Chengdu 610031, China \\ ${ }^{3}$ School of Information Science and Technology, Southwest Jiaotong University, Chengdu 610031, China \\ Correspondence should be addressed to Liang Guo; guoliang@swjtu.edu.cn
}

Received 12 March 2019; Revised 13 May 2019; Accepted 18 June 2019; Published 10 July 2019

Academic Editor: Emiliano Mucchi

Copyright (C) 2019 Liang Guo et al. This is an open access article distributed under the Creative Commons Attribution License, which permits unrestricted use, distribution, and reproduction in any medium, provided the original work is properly cited.

\begin{abstract}
Ball screw, as a crucial component, is widely used in various rotating machines. Its health condition significantly influences the efficiency and position precision of rotating machines. Therefore, it is important to accurately detect faults and estimate fault location in a ball screw system to make sure that the ball screw system runs safely and effectively. However, there are few research studies concerning the topic. The aim of this paper is to fill the gap. In this paper, we propose a method to automatically detect and locate faults in a ball screw system. The proposed method mainly consists of two steps: fault time estimation and instantaneous rotational frequency extraction. In the first step, a statistics-based outlier detection method is proposed to involve the fault information mixing in vibration signals and estimate the fault time. In the second step, a parameterized time-frequency analysis method is utilized to extract the instantaneous rotational frequency of the ball screw system. Once the fault time and instantaneous rotational frequency are estimated, the fault location in a ball screw system is calculated through an integral operation. In order to verify the effectiveness of the proposed method, two fault location experiments under the constant and varying speed conditions are conducted in a ball screw failure simulation testbed. The results demonstrate that the proposed method is able to accurately detect the faults in a ball screw system and estimate the fault location within an error of $22 \%$.
\end{abstract}

\section{Introduction}

Ball screw, a low-friction transmission component providing acceptably smooth motions with relatively high stiffness, is widely used in rotating machines. Due to the tough operation environment and overload, ball screw is one of the most common failure components in rotating machines. Its failure may lead to an equipment breakdown, loss of production and income, and even human casualties $[1,2]$. Therefore, the fault detection and fault location of a ball screw system are important and play a curial role for the reliable operation of rotating machines [3-5].

Although faults of a ball screw system are widespread in rotating machines, to the best of our knowledge, there are limited research reports concerning on health monitoring and fault location of a ball screw system. Jin et al. [6] proposed an intelligent fault diagnosis method to classify multiple failure modes of a ball screw system. Zhang et al. [7] developed a deep learning based condition monitoring system to classify the health condition of a ball screw system. Tsai et al. [8] proposed a technique to determine the onset of preload loss in a ball screw feed drive system via monitoring the change of ball pass frequency. Feng and Pan [9] set up an embedded sensing system for precisely measuring acceleration and temperature of interest points on a ball screw system. This system is able to diagnosis different ball screw preloads through processing acquired signals with further classification using support vector machine method. Han et al. [10] developed a fault diagnosis approach of a ball screw system for determining the running distance at which initial lubrication degradation occurs. Li et al. [11] proposed a systematic methodology for ball screw prognosis to 
implement the fault diagnosis, early diagnosis, health assessment, and remaining useful life prediction. It can be found that the abovementioned research studies mainly focused on some specific failure models classification of a ball screw system and achieved good results. However, there is no research on the fault location.

The fault location of a ball screw system is highly related to its mechanical structure. As shown in Figure 1, a ball screw system consists of a screw shaft, a ball nut, and a lot of balls. The screw shaft provides a helical raceway for the ball nut acting as a precision moving platform, where a ball screw system generates vibration from the interaction forces and friction among balls, a screw, and a nut $[12,13]$. Therefore, the vibration signals acquired from a ball nut are usually applied to detect faults in a ball screw system. As we know, the running position of a ball nut is calculated as follows:

$$
L_{T}=\int_{t=0}^{T} \operatorname{IF}(t) D d_{t},
$$

where $D$ is a screw lead which is one of structure parameters of a ball screw system. $\operatorname{IF}(t)$ is referred to as the instantaneous rotational frequency (IRF), and $T$ is the running time. It should be noted that in the application of fault location, the running time $T$ is the time when a fault is detected, which is also the so-called fault time. Therefore, the fault time and IRF are the two key variations for fault location.

Actually, the core procedure of fault time estimation is the detection of outliers hidden in vibration signals. Concretely, after long-time or overload operation, some defects, such as fatigue and wear, may be found in a ball screw system. When balls roll through those defect regions, the abnormal mechanical vibration characteristics in amplitude and frequency can be detected [13]. Therefore, the intuitive method for ball screw fault detection is outlier detection of vibration signals. Generally, the faults of ball bearings can be detected and localized through the vertical-horizontal synchronization quantitative diagnosis method [14]. Beyond that, the fault detection could be thought of as one problem of outlier detection. Outlier detection refers to the problem of finding points that do not conform to the expected behavior $[15,16]$. One of the simplest methods is $3 \sigma$ principle based outlier detection, which is designed to detect all data instances that are more than $3 \sigma$ distance away from the distribution mean $\mu$. Currently, the $3 \sigma$ principle-based outlier detection methods have been employed in many tasks $[17,18]$.

The IRF extraction has been a hot research topic in recent years, and a large number of reports on this topic have been published. Bonnardot et al. [19] proposed a method for rotating speed reconstruction. In this method, the IRF estimation via vibration signals selected a suitable frequency band around the shaft frequency and then phased demodulating on this band. Combet and Gelman [20] extended the work from Bonnardot et al. [19] and provided some guidelines for the choice of the best band for demodulation. In recent contributions to this area, a promising approach is to use time-frequency representation of vibration signals to extract IRF, since it could produce robust

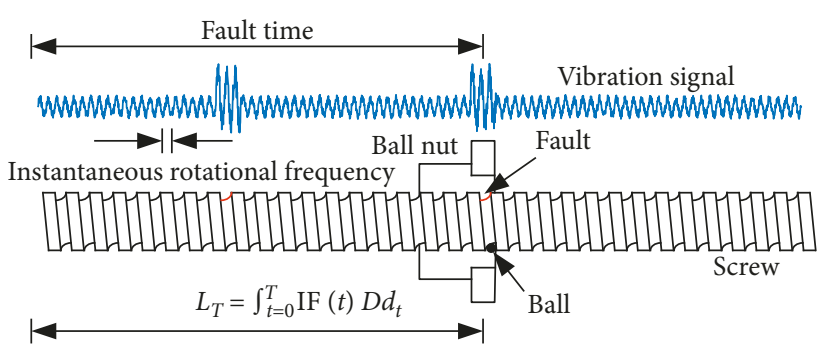

Figure 1: The structure of a ball screw system.

results under noise conditions [21-25]. Typical methods in a broad class of time-frequency analysis include short-time Fourier transfer [26], Wigner-Ville distribution [27], and wavelets [28]. Although those abovementioned nonparameterized time-frequency analysis methods produce promising results and find successful application in IRF extraction, they suffer from a resolution limitation. Alternatively, parameterized time-frequency analysis methods may be more powerful than the nonparameterized ones for IRF extraction since the advantage of improved resolution. The parameterized time-frequency analysis uses some signal dependent parameters to characterize the time-frequency features of the signals to be analyzed. The chirplet transform $[29,30]$ is another kind of linear time-frequency analysis method which is particularly designed for the analysis of chirp-like signals with linear instantaneous frequency. Yang et al. [31-33] proposed a series of parameter estimation procedures for the parameterized time-frequency analysis to analyze nonstationary vibration signals, such as linear transforms-based methods [31], polynomical chirplet transform [32], and spline chirplet transform [33]. Nowadays, those methods have been widely used in instantaneous frequency estimation and received promising results.

Therefore, in this paper, we propose an outlier detection and IRF extraction-based fault detection and location method of a ball screw system. Firstly, using the acquired vibration signals, the ball screw fault and the corresponding fault time are estimated through $3 \sigma$ principle-based outlier detection method, and the IRF of a ball screw system is extracted through a parameterized timefrequency analysis method. Then, based on the estimated fault time and IRF, the fault location is calculated through equation (1). At last, the effectiveness of the proposed method is demonstrated through two constant and varying speed experiments in a designed ball screw failure simulation platform. The main contributions are highlighted as follows:

(1) A $3 \sigma$ principle-based outlier detection method is proposed to detect faults in a ball screw system and estimate the corresponding fault time. Generally, when a ball rolls over the defect section of a ball screw, the amplitude of acquired vibration signals increases. Based on the statistical characteristics, a point whose amplitude exceeds a predefined $3 \sigma$ threshold can be recognized as an outlier one. The corresponding outlier time is recognized as the fault time. 
(2) A fault location method of a ball screw system based on the outlier detection and IRF extraction is proposed. In this method, the IRF is extracted through parameterized time-frequency analysis, and the fault time is estimated through the $3 \sigma$ principle-based outlier detection method. The result of IRF integration under the fault time is the fault location of a ball screw system.

(3) In the designed experiment testbed, two fault location experiments under the constant and varying speed condition are conducted. The experimental results demonstrate the effectiveness of the proposed method for fault detection and location of a ball screw system.

The rest of the paper is organized as follows. Section 2 introduces the parameterized time-frequency analysis method. The detailed procedure of the proposed method is presented in Section 3. Using the designed experiment platform, the performance of the proposed method is evaluated in Section 4. Finally, conclusions are drawn in Section 5 .

\section{Preliminary}

A polynomial chirplet transform is a modified version of a conventional chirplet transform [34]. The conventional chirplet transform is an inner product between an input signal and chirplets. The chirplet is a series of window transform of a chirp function, where the window transform provides a time domain localization property. In a timefrequency space, chirplets exist as rotated, sheared, or other structures moving from the traditional parallelism [35]. Generally, in the time-frequency analysis, the modules are of interest. Therefore, the conventional chirplet transform can be written as

$$
C_{x}\left(t_{0}, \omega, \alpha ; \sigma\right)=\int_{-\infty}^{+\infty} z(t) \Phi_{\alpha}^{R}(t) \Phi_{\alpha}^{S}\left(t, t_{0}\right) \omega_{\alpha}\left(t-t_{0}\right) e^{(-j \omega t)} d t,
$$

where $z(t)$ is the analytical signal of input signal $x(t)$, generated by the Hilbert transform, i.e., $z(t)=x(t)+$ $j H[x(t)] ; \Phi_{\alpha}^{R}(t)=e^{\left(-\left(j \alpha t^{2} / 2\right)\right)}$ rotates the analytical signal $z(t)$ by an angle $\theta$ with $\operatorname{tg}(\theta)=-\alpha$; and $\Phi_{\alpha}^{S}\left(t, t_{0}\right)=e^{\left(j \alpha t_{0} t\right)}$ is the frequency shift operation.

In the convolutional chirplet transform, when a chirp rate is properly selected, the chirplet transform would render the time-frequency distributions of an excellent concentration. However, in the real-world application, the instantaneous frequency of an input signal may not be exactly a linear function of time. In that situation, the convolutional chirplet transform faces the limitation for tracking the evolution versus time of the instantaneous frequency of the input signal. Therefore, in order to improve the efficacy of the convolutional chirplet transform in analyzing the signals with a nonlinear instantaneous frequency trajectory, a modified version known as the polynomial chirplet transform is used, which is defined as follows [27]:

$$
\begin{aligned}
\operatorname{PCT}_{x}\left(t_{0}, \omega, \alpha_{1}, \ldots, \alpha_{n} ; \sigma\right)= & \int z(t) \Phi_{\alpha_{1}, \ldots, \alpha_{n}}^{R} \Phi_{\alpha_{1}, \ldots, \alpha_{n}}^{S}\left(t, t_{0}\right) \\
& \times \omega_{\sigma}\left(t-t_{0}\right) e^{-j \Omega t} d t
\end{aligned}
$$

where $z(t)$ is the analytical signal of input signal $x(t)$; $\Phi_{\alpha_{1}, \ldots, \alpha_{n}}^{R}(t)$ and $\Phi_{\alpha_{1}, \ldots, \alpha_{n}}^{S}\left(t, t_{0}\right)$ are the nonlinear frequency rotating and frequency shift operator, respectively; $\omega_{\sigma}\left(t-t_{0}\right)$ is the window function; and $\sigma$ is the size of the window. Moreover, the $\Phi_{\alpha_{1}, \ldots, \alpha_{n}}^{R}(t)$ and $\Phi_{\alpha_{1}, \ldots, \alpha_{n}}^{S}\left(t, t_{0}\right)$ are defined as follows:

$$
\begin{aligned}
\Phi_{\alpha_{1}, \ldots, \alpha_{n}}^{R}(t) & =e^{-j \sum_{k=2 \pi}^{n+1} \alpha_{k-1} t^{k}}, \\
\Phi_{\alpha_{1}, \ldots, \alpha_{n}}^{S}\left(t, t_{0}\right) & =e^{j \sum_{k=2}^{n+1} \alpha_{k-1} 1_{0}^{k-1} t},
\end{aligned}
$$

where $\left(\alpha_{1}, \ldots, \alpha_{n}\right)$ are the polynomial kernel characteristic parameters. Suppose that $z(t)$ is the consideration signal. First, $z(t)$ is rotated by $\sum_{k=2}^{n+1}(1 / k) \alpha_{k-1} t^{k-1}$. Then, it is shifted by $\sum_{k=2}^{n+1} \alpha_{k-1} t_{0}^{k-1} t$. At last, the $\operatorname{IF}_{x}\left(t_{0}, \sigma\right)$ is calculated through short-time Fourier transfer at the time span $t_{0}-(\sigma / 2)$ and $t_{0}+(\sigma / 2)$.

Once the polynomial kernel characteristic parameters are given, the polynomial chirplet transform could produce a high-quality time-frequency distribution of the considerable signal. Suppose that the polynomial chirplet transform $\mathrm{PCT}_{x}$ of signal $x(t)$ is referred to as time-frequency distribution $\mathrm{TFD}_{x}$. The main frequency component in the signal $x(t)$ is obtained through locating the peak value of $\operatorname{TFD}_{x}$ at the time $t_{0}$ :

$$
\operatorname{IF}\left(t_{0}\right)=\max \left(\operatorname{TFD}_{x}\left(t_{0}\right)\right),
$$

where $\operatorname{IF}\left(t_{0}\right)$ is the instantaneous main frequency of signal $x$ at the time $t_{0}$ and $\max (\cdot)$ is the maximum operation.

\section{Proposed Method}

The main purpose of the proposed method is to locate faults appearing in a ball screw system. According to equation (1), once the fault time and IRF are estimated, the fault locations can be easily calculated. Therefore, as shown in Figure 2, the proposed method mainly consists of three steps: data acquisition, fault time estimation and IRF extraction, and fault location. In the step of data acquisition, an accelerometer is installed in a nut which moves along a screw. In consequence, the acquired vibration signal is able to monitor the condition of a ball screw system. Then, in the stage of fault time estimation and IRF extraction, the fault time and IRF information are all extracted from the acquired vibration signals. This stage is the crucial one for the proposed method, and we detail it as follows.

3.1. Fault Detection and Fault Time Estimation. When balls roll over fault defect regions of a ball screw system, the unstable contact between balls and a screw may produce an impact. The impact results in outliers hide in monitoring vibration signals. Therefore, a $3 \sigma$ principle-based outlier 


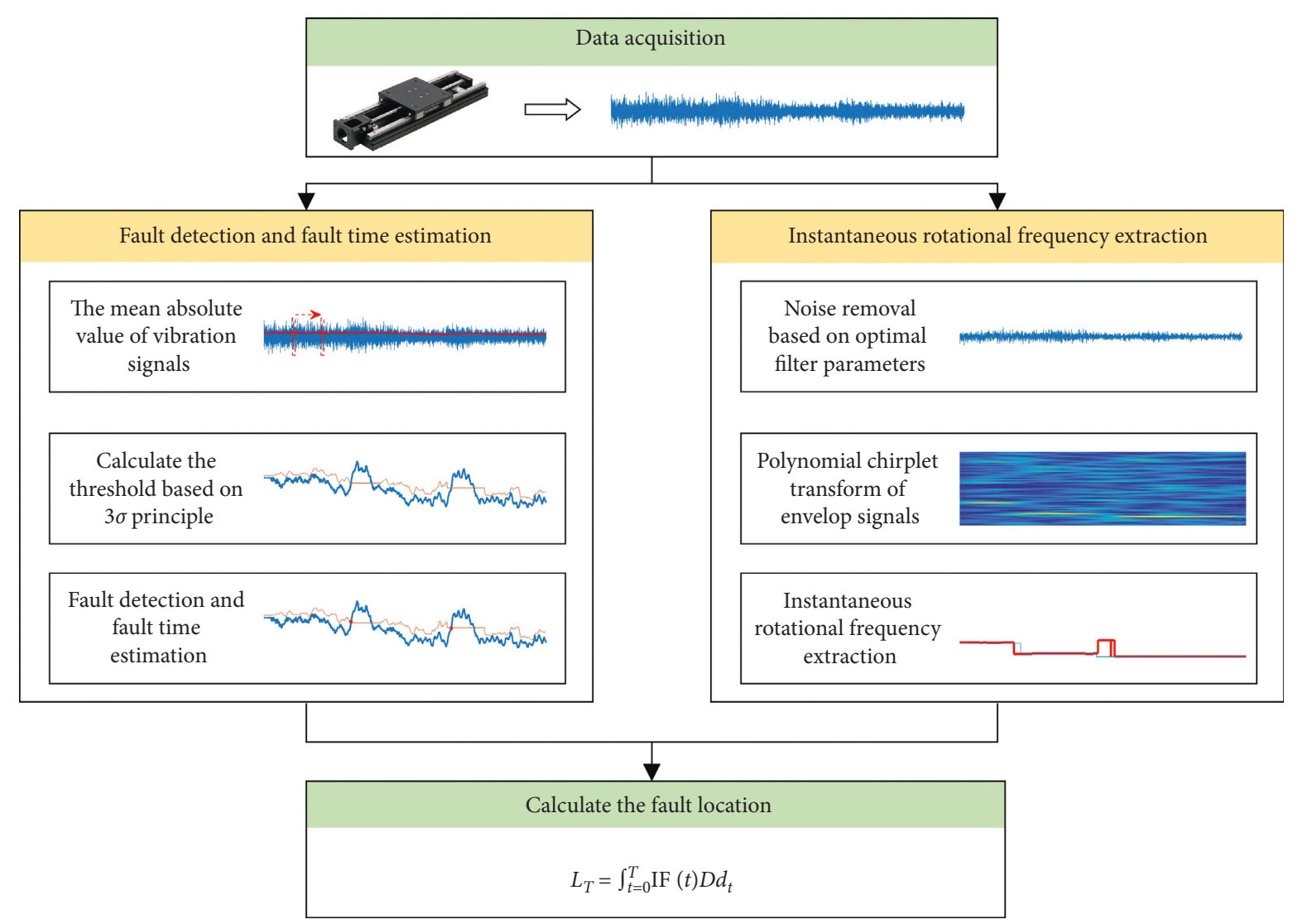

Figure 2: Flowchart of the proposed method.

detection method is proposed in this paper to detect outlier region in the monitoring vibration signal to find out faults in a ball screw system and estimate corresponding fault time. The detailed flowchart of the proposed fault detection and fault time estimation method is shown in Figure 3. Moreover, the whole procedure mainly includes three substeps, which are detailed in the following.

3.1.1. Data Preprocessing. The acquired raw vibration signals are with noises. Those noises may submerge the characteristic component caused by faults. Therefore, a data preprocessing operation, i.e., the mean absolute value of the segmented vibration signal, is conducted to produce a smoothed signal as follows:

$$
\bar{x}_{i}=\frac{1}{N} \sum_{j=i}^{i+N}\left|x_{j}\right|,
$$

where $\bar{x}_{i}$ is the smoothed signal, $x_{j}$ is the raw vibration signal, and $N$ is the smooth length. $|\cdot|$ calculates an absolute value.

3.1.2. Fault Detection. When balls roll through faulty regions located in a screw, the amplitude of acquired vibration signals usually get large. The large amplitude is able to recognize outliers. Therefore, a $3 \sigma$ rule-based outlier detection method is presented to detect outliers in the smoothed signal $\bar{x}$. In the method, a threshold Thr is first set up as follows:

$$
\text { Thr }=\mu+3 \sigma \text {, }
$$

where $\mu$ and $\sigma$ are the expectation and deviation of the signal $\bar{x}$, respectively. A point that exceeds the failure threshold is defined as the outlier point.

3.1.3. Fault Time Estimation. Once the outliers are detected, the fault time $T$ is able to be estimated as follows:

$$
T=t_{\text {fault }}-t_{\text {start }},
$$

where $t_{\text {fault }}$ is the time detected outliers and $t_{\text {start }}$ is the operation starting time.

3.2. Instantaneous Rotational Frequency Extraction. The above step estimates the fault time. In this step, we set up a method to extract IRF from the acquired vibration signals. The flowchart of the IRF extraction is shown in Figure 4. Additionally, the detailed procedure is concluded as the following three steps.

3.2.1. Data Preprocessing. The spectral kurtosis (SK) of filtered signals in different plane $(f, \Delta f)$ is calculated using equation (9). The plane $(f, \Delta f)$ with the maximum SK value 


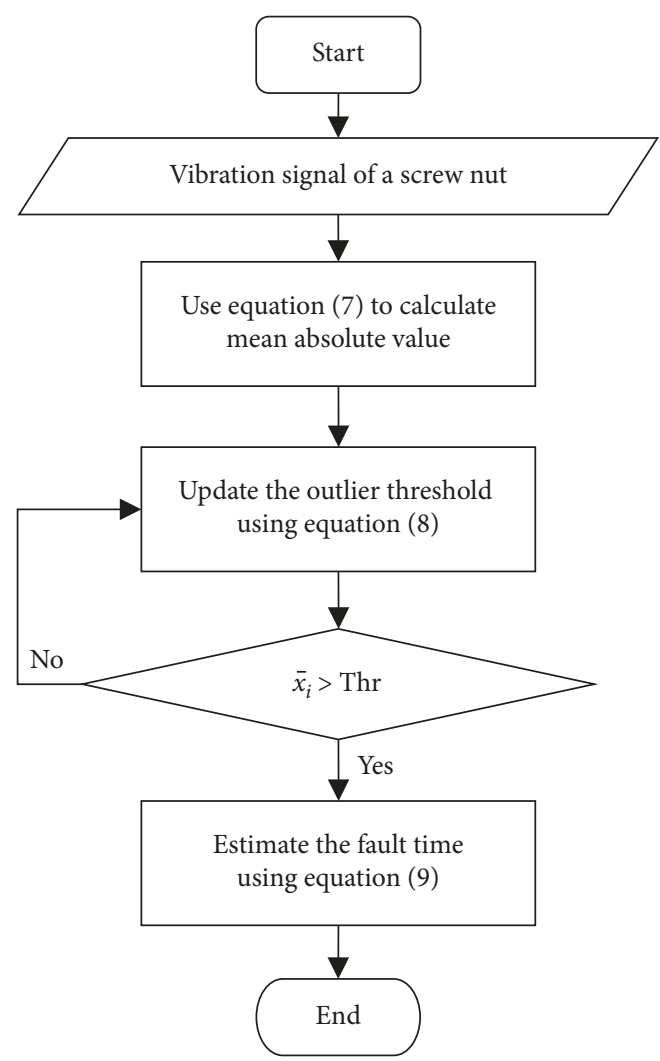

FIGURE 3: Flowchart of the proposed fault detection and fault time estimation method.

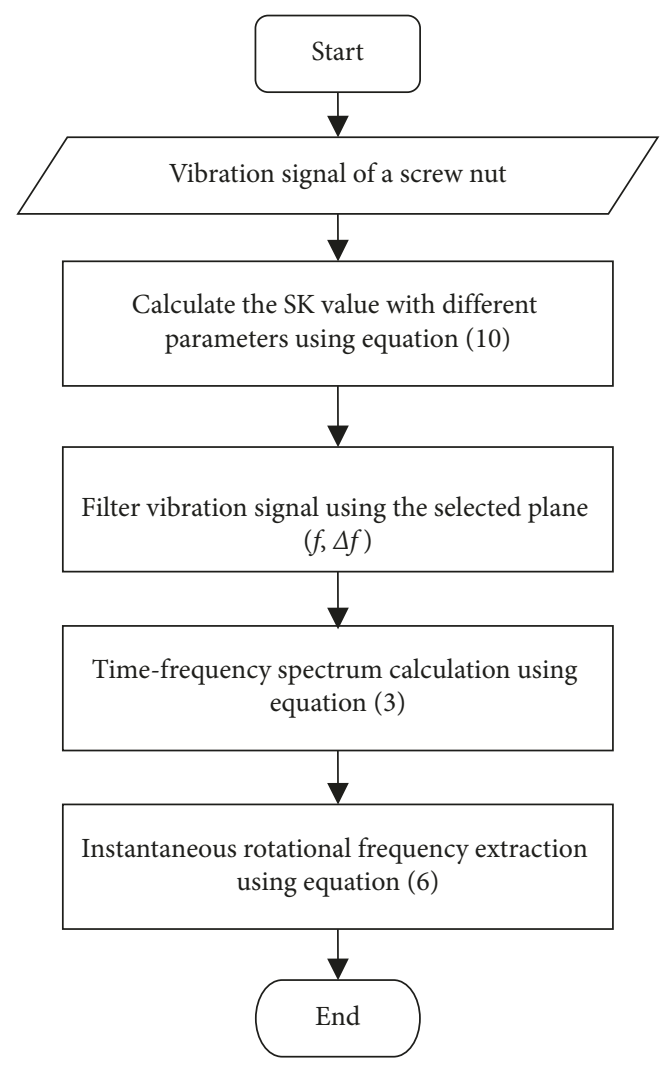

FIGURE 4: Flowchart of the proposed instantaneous rotational frequency extraction method. is chosen as the bandpass filter parameters to preprocess an acquired signal $x$.

$$
K_{k}^{i}=\frac{\left\langle\left|c_{k}^{i}(n)\right|^{4}\right\rangle}{\left\langle\left|c_{k}^{i}(n)\right|^{2}\right\rangle^{2}}-2,
$$

where $c_{k}^{i}(n)$ be the sequence of coefficients issued from the $i$ th filter, $i=0, \ldots, 2^{k}-1$, at the $k$ th level in the decomposition tree.

3.2.2. Time-Frequency Analysis. The filtered signals are demodulated through Hilbert transform to obtain the envelope signals. The envelop signals are transferred into a time-frequency spectrum through polynomial chirplet transform. The amplitude of time-frequency spectrum is calculated using equation (3).

3.2.3. IRF Estimation. Based on the theoretical rotational frequency $f_{\max }$ of a system, the maximum value in a frequency range $\left[0, f_{\max }\right]$ is extracted as the IRF. The maximum value is calculated using equation (5).

Once the fault time and IRS are estimated, the fault location is calculated using equation (1).

\section{Experiment and Discussion}

4.1. Experiment Data. An experiment platform, as shown in Figure 5, is designed to conduct some condition monitoring and fault location experiments of a ball screw system. This platform mainly contains two parts: a mechanical motion part and a data acquisition part. The mechanical motion part includes a synchronous motor with a ball screw and two supporting bearings. A synchronous motor acts as the actuator that allows the screw to rotate through two supporting bearings and a screw nut. In order to monitor the operation condition of the ball screw system, three accelerometers are mounted. They are located in the two supporting bearings and the screw nut, respectively. A Beckhoff controller-based data acquisition card is installed to collect the vibration signals. The Beckhoff controller enables the motion control and data acquisition to be integration.

In the experiment, the test ball screw is SFU2005-4, and the detailed information is documented in Table 1 . To verify the proposed fault detection and location method, two faults are simulated on the screw through an electrical discharge machining. As shown in Figure 5, the two simulated faults are located at $90 \mathrm{~mm}$ and $160 \mathrm{~mm}$, respectively. Then, three different experiments are conducted. The speed of the first two experiments is set as piecewise static and that of the third experiment is set as dynamic. In the first experiment, the driven motor rotates with a constant speed $180 \mathrm{rpm}$, i.e. the rotational frequency is $3.00 \mathrm{~Hz}$. In the second experiment, the driven motor rotates with the fluctuation speed $225 \mathrm{rpm}$, $120 \mathrm{rpm}$, and $90 \mathrm{rpm}$, i.e., the rotational frequency is $3.75 \mathrm{~Hz}$, $2.00 \mathrm{~Hz}$, and $1.50 \mathrm{~Hz}$, respectively. In those two experiments, the vibration signals at the screw nut are acquired and shown in Figures 6(a) and 6(b), respectively. 


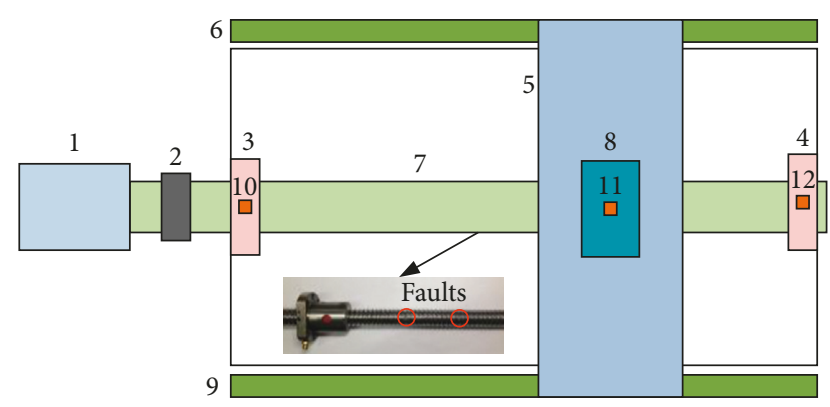
(1) Motor
(5) Motion platform
(9) Guide rail
(2) Couple
(6) Guide rail
(10) Accelerometer
(3) Support bearing
(7) Screw
(11) Accelerometer
(4) Support bearing
(8) Ball nut
(12) Accelerometer

FIGURE 5: The designed experiment platform. 1: motor; 2: couple; 3: support bearing; 4: support bearing; 5: motion platform; 6: guide rail; 7: screw; 8: ball nut; 9: guide rail; 10: accelerometer; 11: accelerometer; 12: accelerometer.

TABle 1: Parameters of the test ball screw.

\begin{tabular}{|c|c|c|}
\hline \multicolumn{3}{|c|}{ SFU2005-4 } \\
\hline Nominal diameter $(\mathrm{mm})$ & $\begin{array}{l}\text { Nominal lead } \\
(\mathrm{mm})\end{array}$ & $\begin{array}{l}\text { Ball diameter } \\
(\mathrm{mm})\end{array}$ \\
\hline 20 & 5 & 3.17 \\
\hline
\end{tabular}

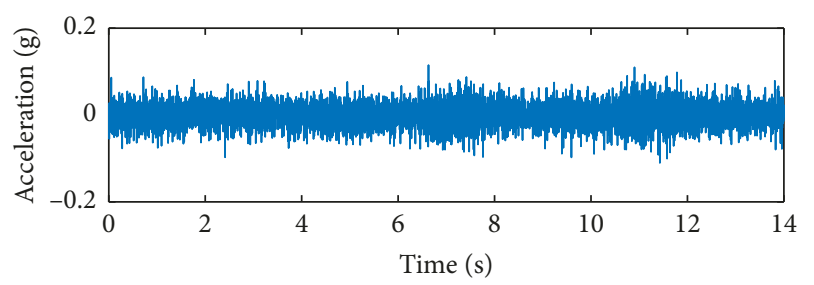

(a)

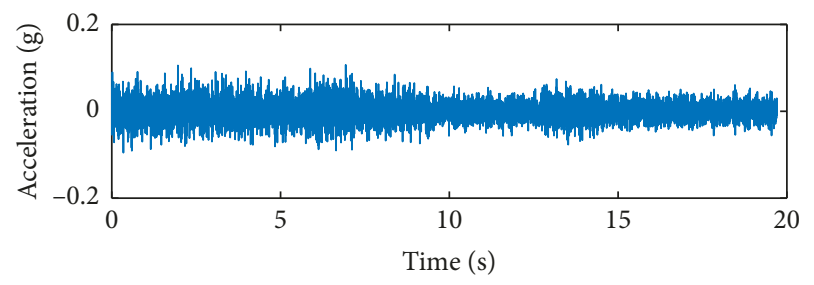

(b)

FIGURE 6: The vibration signals of two experiments. (a) Experiment 1. (b) Experiment 2.

4.2. Fault Time Estimation. In order to validate the effectiveness of the proposed fault time estimation method, a simulated case study is first conducted. In the simulated case, different signals with various fault induced impulse are utilized. The simulation signal is constructed by the following equation:

$$
x(t)=A e^{-\beta\left(t / f_{s}\right)} \sin \left(2 \pi f\left(\frac{t}{f_{s}}\right)+\tau\right)+v(t),
$$

where $A$ is the amplitude that is set as 1 . The damping factor $\beta$ equals 500. The sampling frequency $f_{s}$ and oscillating frequency $f$ are set as $20000 \mathrm{~Hz}$ and $2000 \mathrm{~Hz}$, respectively. $\tau$ is the phase position of the carrier wave. $v(t)$ is the Gaussian white noise with a signal-to-noise ratio $-2 \mathrm{~dB}$. Meanwhile, a fault-induced impulse component is added into the simulation signal, as shown in Figure 7(a).

In the experiment, the amplitude of the fault-induced impulse component is $N$ times the amplitude of the simulation signal. It should be noted that although there are some impulse components in the acquired vibration signals, they are not totally separable. Therefore, we first enhance those impulse components through calculating the mean absolute value of the segmented acquisition signals. Figure $7(\mathrm{~b})$ is the result of outlier detection where the amplitude of the fault induced impulse component is 1.2 times the amplitude of the simulation signal. From the figure, it can be observed that the proposed method is able to detect the outliers in the signal. In the experiment, $N$ is further set to range from 1.1 to 2.0. The estimated time of outliers is documented in Table 2. Those results also demonstrate the power of the proposed method for detecting outliers and estimating fault time.

The effectiveness of the proposed fault time estimation method has been demonstrated through the above simulated case study. Now, the method is applied to process the acquired signals shown in Figure 6. The impulse components in the acquired signals are also first enhanced through the mean absolute value method. The mean window, i.e., the red window as shown in Figure 7 , shifts as the time axial to obtain the smoothed curve. In this paper, the length of the mean window is set to 1000 . The results of the smoothed signals are the blue line in Figure 8. It can be found that in the smoothed vibration signals, the impulse components are more salient and the noise is basically eliminated. This allows us to easily detect faults.

Then, we detect faults and estimate the fault time using the $3 \sigma$ principle-based outlier detection method. The results are presented in Figure 8. In the figure, the orange line is the failure threshold calculating through equation (7), and the red points are the detected outliers which are also the fault points. The estimated fault time are documented in Table 3.

4.3. Instantaneous Rotational Frequency Estimation. As the procedure of the proposed method as shown in Figure 4, we extract the IRF of the test ball screw system as the following steps. Firstly, the optimal bandpass filter parameters are selected. Depending on the length of signals, the maximum decomposition level is set to 5 , the spectral kurtosis of different filter parameters is calculated, and the results are plotted in Figure 9. For the acquired signals in the constant speed, the optimal filter parameter is a center frequency $f_{c}=$ $62.5 \mathrm{~Hz}$ with a bandwidth $B_{w}=125 \mathrm{~Hz}$. For the signals in the varying speed, the optimal filter parameter is a center frequency $f_{c}=20.8 \mathrm{~Hz}$ with a bandwidth $B_{w}=41.7 \mathrm{~Hz}$.

Once obtaining those optimal filter parameters, the acquired vibration signals are filtered using the bandpass filter, and the results are shown in Figure 10. From those 


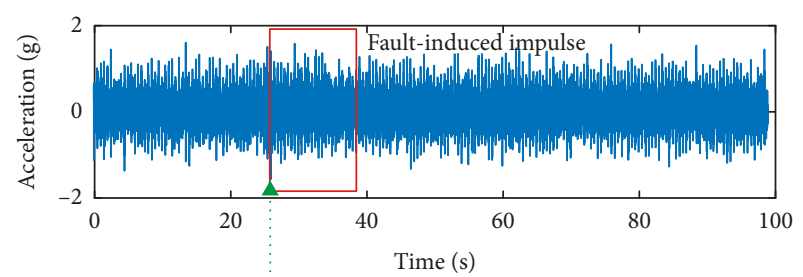

(a)

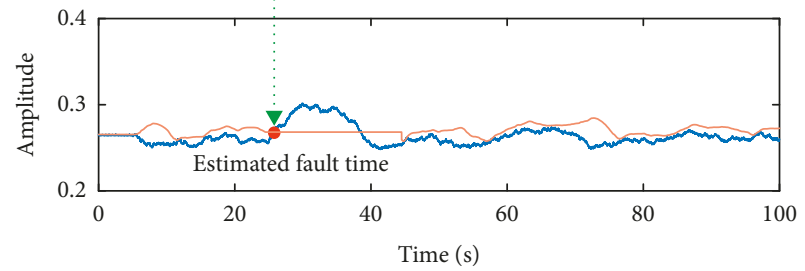

(b)

FIgURE 7: The results of a simulated case study: (a) original signal; (b) fault detection and fault time estimation.

TABLE 2: Fault time estimation results.

\begin{tabular}{lcccccc}
\hline & \multirow{5}{*}{ Real } & \multicolumn{5}{c}{ Estimation of different $N$} \\
& & 1.1 & 1.2 & 1.5 & 1.8 & 2.0 \\
\hline Time (s) & 25 & 22.7 & 25.8 & 25.5 & 25.1 & 24.8 \\
Error rate (\%) & & 9.2 & 3.2 & 2.0 & 0.4 & 0.8 \\
\hline
\end{tabular}

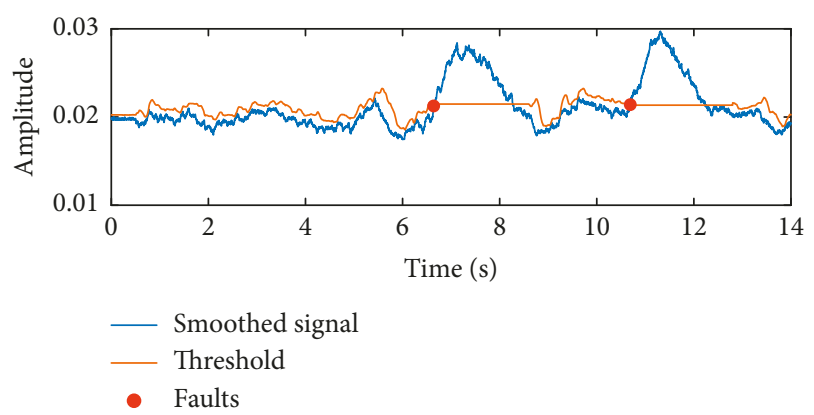

(a)

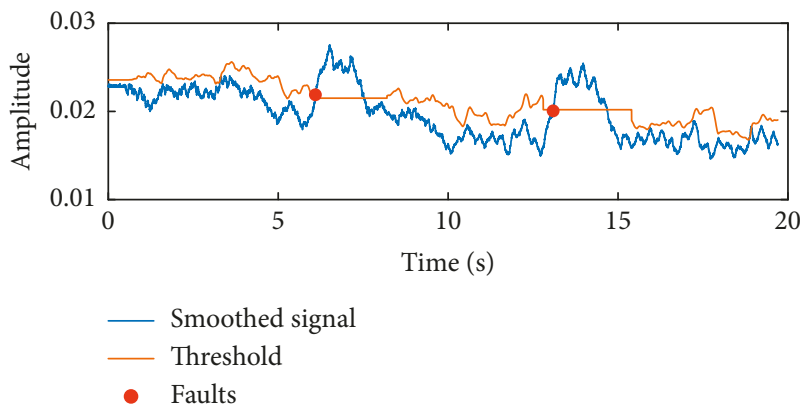

(b)

Figure 8: Results of the fault detection: (a) experiment 1 and (b) experiment 2 .

figures, the noise hidden in the periodic signals are basically removed, which allows the IRF estimation to be easier. Finally, the polynomial chirplet transform is applied to obtain the time-frequency spectrum of the filtered signals.
TABLE 3: Fault time of the two experiments.

\begin{tabular}{lcc}
\hline Experiments & The first fault time (s) & The second fault time (s) \\
\hline 1 & 6.64 & 10.69 \\
2 & 6.09 & 13.09 \\
\hline
\end{tabular}

The time-frequency distribution is shown in Figure 11. Figure 11(b) is the detailed exhibition of Figure 11(a) in the frequency span $[0,12.5]$ which is decided by the rated speed of the driven motor applied in the experiment platform, in which the IRF and its harmonic curves are clearly exhibited. According to equation (5), the IRF of the constant speed is calculated and shown in Figure 11(c), i.e., the red line. Compared to the real IRF, i.e., the blue line in Figure 11(c), the estimated IRF is almost stationary and equals to the true IRF. Figure $11(\mathrm{~d})$ is the result of vibration signals from the varying speed experiment. Also, its processing procedures are same as the constant speed experiment. In Figure 11(f), it can be found that the estimated IRF is mixed and unstable at the junction points of different rotating speeds, and this may be caused by the length of data truncation window in equation (8). Although the estimated IRF is unstable at the junction points, it generally remains stable at the condition of the constant speed.

In order to validate the superiority of the proposed method, a comparison experiment is conducted. In the experiment, a commonly used time-frequency analysis technique, continuous wavelet transform (CWT) [32], is applied to compare with the method in the paper. In the experiment, the comr3-3 in MATLAB is applied to process the acquired vibration signal. From Figure 12, it can be seen that the extracted IRF based on CWT presents to be obvious fluctuation. It seems that the smoothness and stability of the CWT-based method are worse than those of the proposed method. The fluctuation and instability may affect the estimation of IRF and fault location. The comparison experiment indicates that the proposed method presents the superiority for the IRF estimation.

4.4. Dynamic Speed Estimation. The speed of the above two experiments is set as piecewise static without fluctuations. However, in the industrial application, the speed of a ball screw may be dynamic. Therefore, in order to further validate the effectiveness of the proposed method, a dynamic speed experiment is conducted. In the experiment, the speed of the testing ball screw varies with the running time. The raw vibration signal of this experiment is shown in Figure 13(a). Firstly, in the step of fault time estimation, the length of the mean window is also set to 1000 . The fault points of the smoothed signals are detected as shown in Figure 13(a). The first and second fault time is estimated as $7.5 \mathrm{~s}$ and $11.2 \mathrm{~s}$, respectively. Secondly, in the step of IRF estimation, the vibration signal is filtered through the center frequency with the bandwidth. The time-frequency spectrum of the vibration signal is calculated with the polynomial chirplet transform, as indicated in Figure 14(a). At last, the IRF can be estimated through equation (5), as shown in Figure 14(b). 


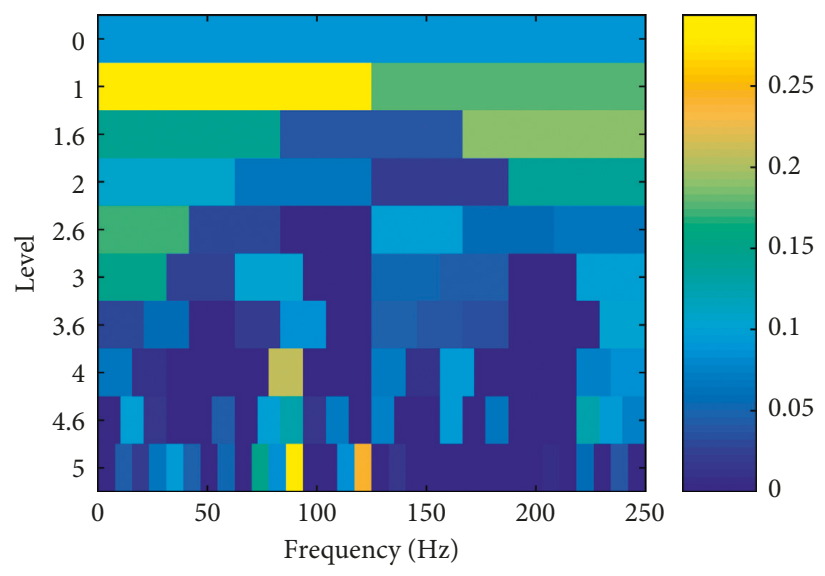

(a)

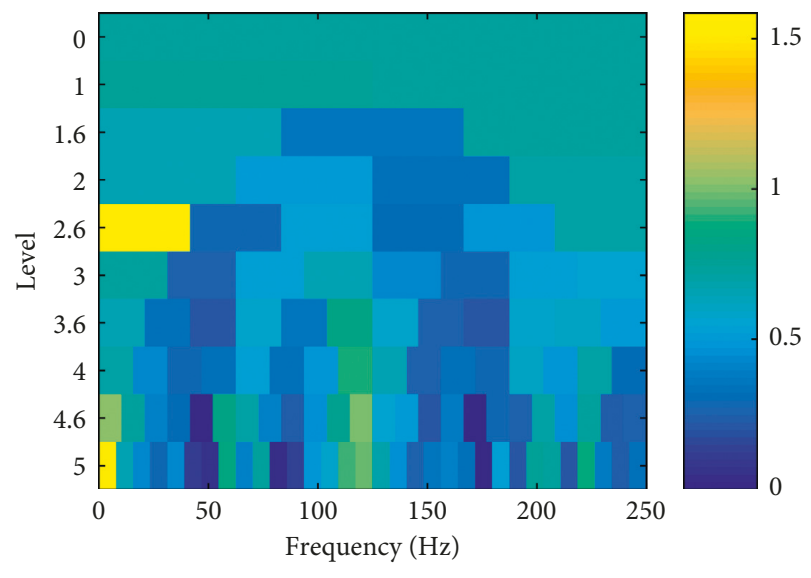

(b)

FIgURE 9: Results of SK of the two vibration signals. (a) Experiment 1: $B_{w}=125 \mathrm{~Hz} ; f_{c}=62.5 \mathrm{~Hz}$. (b) Experiment $2: B_{w}=41.6667 \mathrm{~Hz}$; $f_{c}=20.8333 \mathrm{~Hz}$.

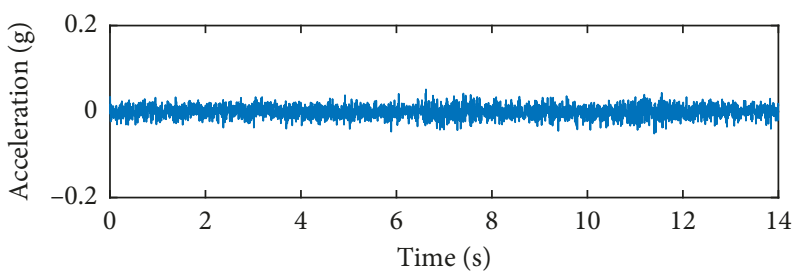

(a)

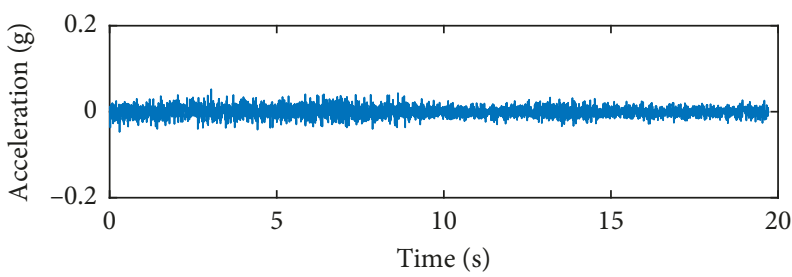

(b)

Figure 10: The filtered signals: (a) experiment 1 and (b) experiment 2.

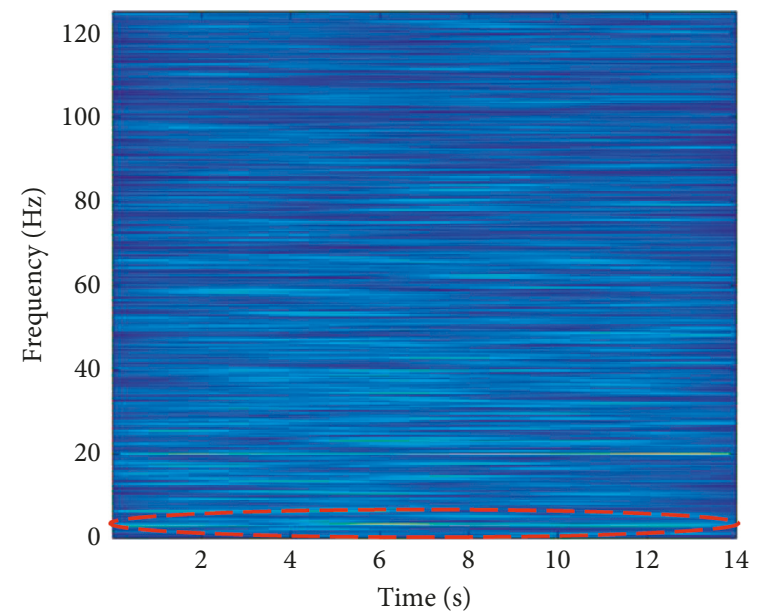

(a)

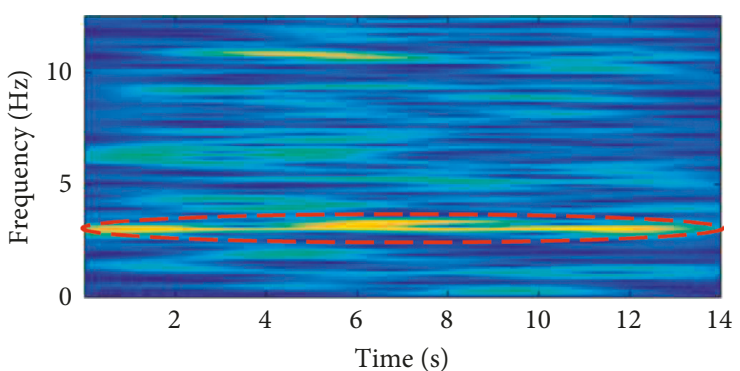

(b)

FIgURE 11: Continued. 

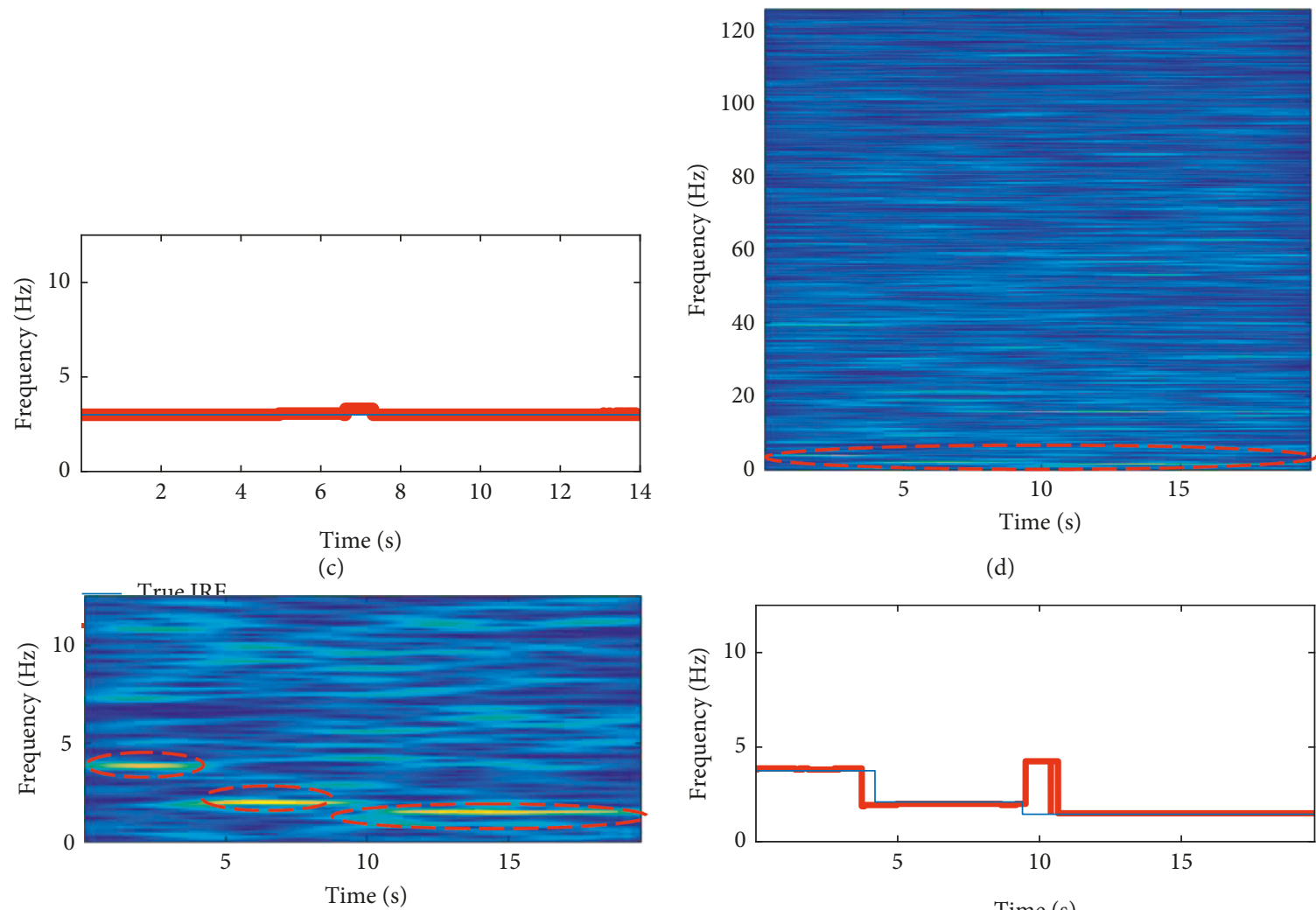

(d)

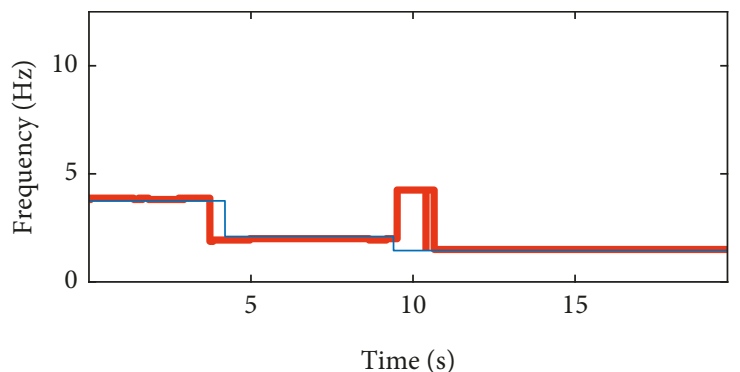

- True IRF

_ Estimated IRF

(e)

(f)

Figure 11: The results of the instantaneous rotational frequency estimation: (a) the time-frequency distribution of experiment 1; (b) the detailed time-frequency distribution of experiment 1 ; (c) the instantaneous rotational frequency of experiment 1 ; (d) the time-frequency distribution of experiment 2; (e) the detailed time-frequency distribution of experiment 2; (f) the instantaneous rotational frequency of experiment 2 .

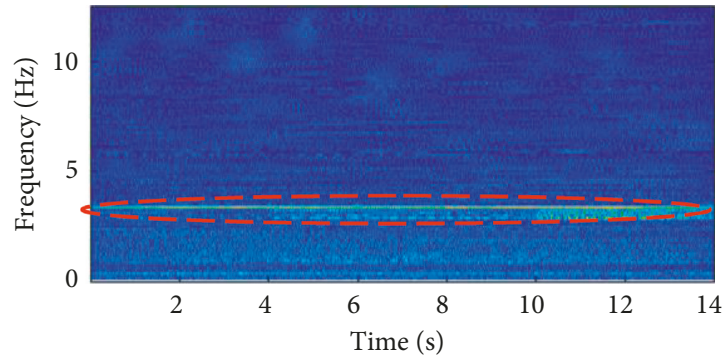

(a)

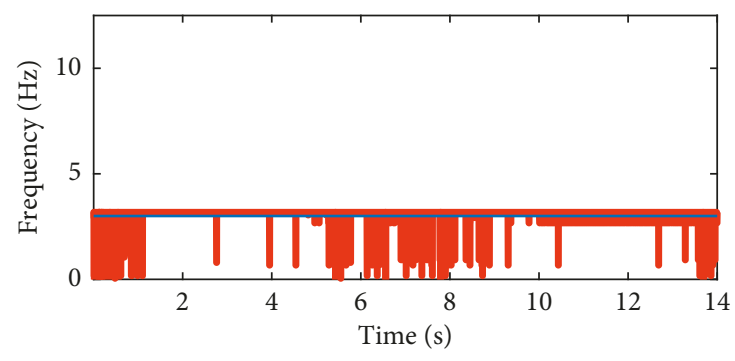

True IRF

Estimated IRF

(b)

Figure 12: Continued. 


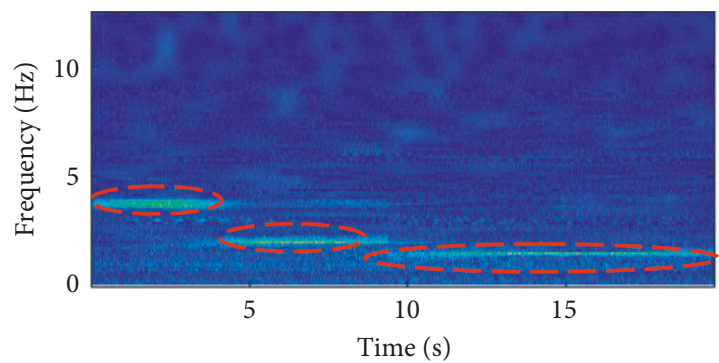

(c)

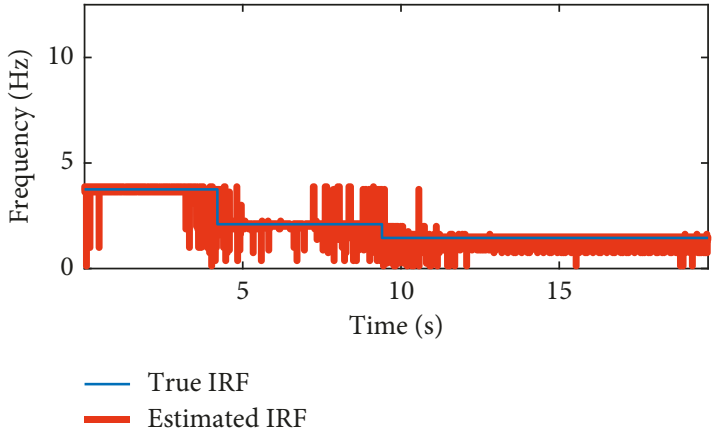

(d)

FIGURE 12: The results of the instantaneous rotational frequency estimation based on continuous wavelet transform: (a) the detailed timefrequency distribution of experiment 1; (b) the instantaneous rotational frequency of experiment 1 ; (c) the detailed time-frequency distribution of experiment 2; (d) the instantaneous rotational frequency of experiment 2.

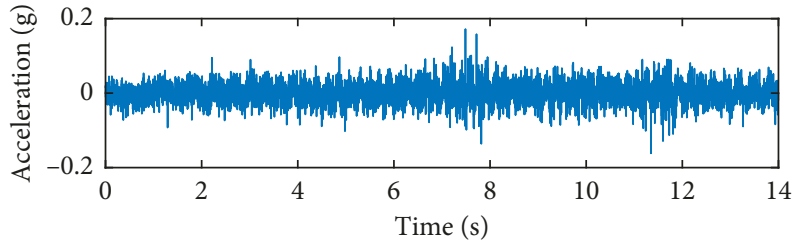

(a)

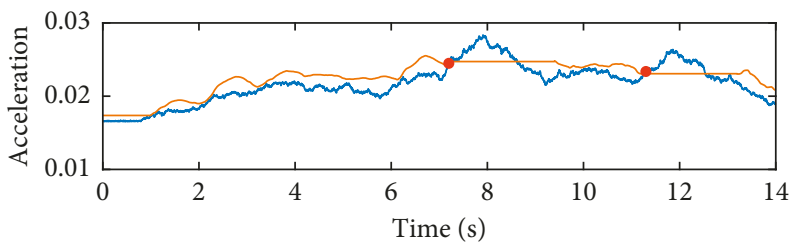

(b)

Figure 13: Vibration signal and 3 sigma outlier detection: (a) vibration signal; (b) outlier detection.

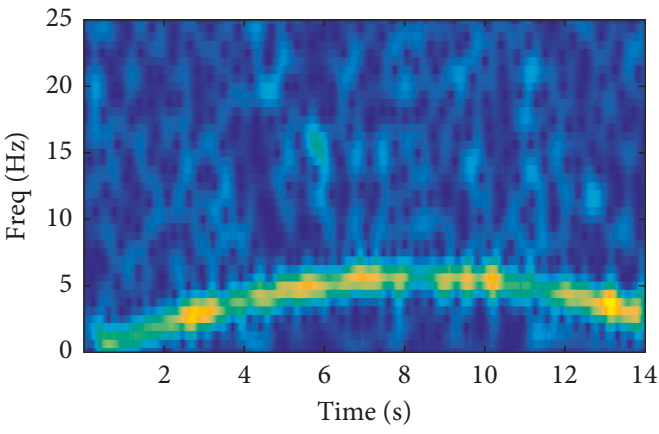

(a)

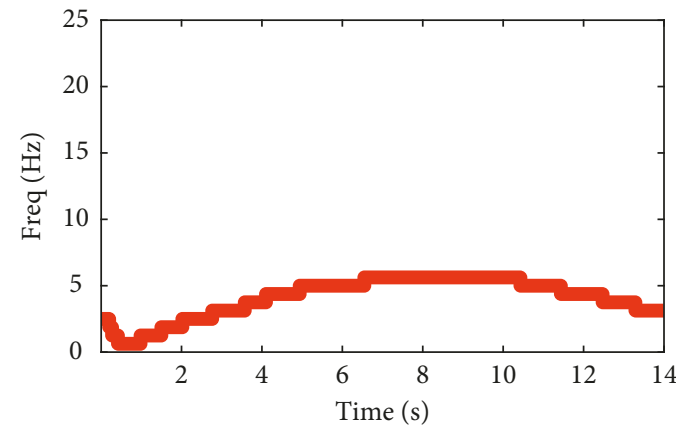

(b)

FIGURE 14: Instantaneous rotational frequency estimation: (a) time-frequency analysis and (b) instantaneous rotational frequency.

4.5. Fault Location. After the fault time $T$ and $\operatorname{IRF} \operatorname{IF}(t)$ are obtained, the fault location of the ball screw system is calculated through equation (1), where the screw lead $D$ in the experiments is $5 \mathrm{~mm}$. The estimation results are documented in Table 4. In order to further assess those results, the relative error is calculated as follows:

$$
R_{e}=\frac{\left|P_{r}-P_{p}\right|}{\left|P_{r}\right|} \times 100 \%,
$$

where $P_{r}$ is the real position and $P_{p}$ is the estimated position, and the relative errors are shown in Table 4 . For comparison,
TABLE 4: Fault location results.

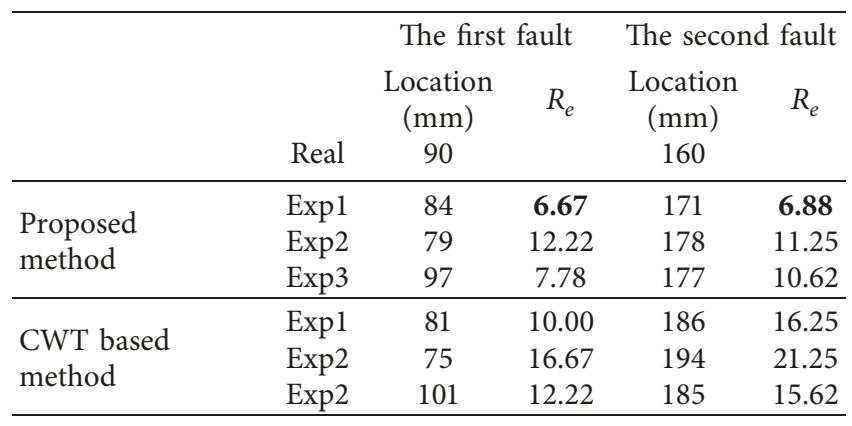


the CWT-based fault location estimation results are also documented in Table 4 which shows that the CWT-based method produces a lower accuracy than the proposed method.

Furthermore, from Table 4, it is seen that the relation error at the constant speed experiment is much smaller than the one at the varying speed experiment. That is the reason of dynamic accumulation error. Additionally, the relation error results of the three experiments are less than $22 \%$, and it indicates that the proposed method is able to locate the faults in the ball screw system within a small error. This enables the proposed method to be potentially applied to the industrial field.

\section{Conclusions}

This paper proposes a ball screw fault detection and location method. Firstly, we set up a $3 \sigma$ principle-based fault detection method, which allows us to discover faults of a ball screw system and calculate the fault time. A simulated case study verifies that the proposed $3 \sigma$ principle-based fault detection method is able to detect faults under various compact levels. Then, the polynomial chirplet transform is utilized to estimate the IRF of the test ball screw, and the polynomial chirplet transform-based method produces a better result than the CWT-based method for the IRF estimation. Combining the estimated fault time with the extracted IRF, fault locations of the ball screw system are calculated. In order to verify the effectiveness of the proposed method, an experiment platform of the ball screw system is designed. In the experiment platform, two experiments, i.e., the constant speed and the varying speed, are conducted to test the proposed fault detection and location method. The results demonstrate that the proposed method is able to detect faults effectively and locate faults within a small error.

\section{Data Availability}

The experiments are conducted with the support of a company. The company does not want to make the experiment data public currently.

\section{Conflicts of Interest}

The authors declare that they have no conflicts of interest.

\section{Acknowledgments}

This research was supported by the National Natural Science Foundation of China (no. 51775452) and Fundamental Research Funds for the Central Universities (2682019CX35 and 2018GF02).

\section{References}

[1] Y. Lei, Intelligent Fault Diagnosis and Remaining Useful Life Prediction of Rotating Machinery, Butterworth-Heinemann, Oxford, UK, 2016.

[2] L. Guo, N. Li, F. Jia, Y. Lei, and J. Lin, "A recurrent neural network based health indicator for remaining useful life prediction of bearings," Neurocomputing, vol. 240, pp. 98-109, 2017.
[3] Z. Wang, J. Zhou, J. Wang et al., "A novel fault diagnosis method of gearbox based on maximum kurtosis spectral entropy deconvolution," IEEE Access, vol. 7, pp. 29520-29532, 2019.

[4] E. A. Dias, F. B. Pereira, S. L. M. Ribeiro Filho, and L. C. Brandão, "Monitoring of through-feed centreless grinding processes with acoustic emission signals," Measurement, vol. 94, pp. 71-79, 2016.

[5] J. Liu and Y. Shao, "Dynamic modeling for rigid rotor bearing systems with a localized defect considering additional deformations at the sharp edges," Journal of Sound and Vibration, vol. 398, no. 23, pp. 84-102, 2017.

[6] W. Jin, Y. Chen, and J. Lee, "Methodology for ball screw component health assessment and failure analysis," in Proceedings of the ASME 2013 International Manufacturing Science and Engineering Conference Collocated with the 41st North American Manufacturing Research Conference, American Society of Mechanical Engineers (ASME), Madison, WI, USA, June 2013.

[7] L. Zhang, H. Gao, J. Wen, S. Li, and Q. Liu, “A deep learningbased recognition method for degradation monitoring of ball screw with multi-sensor data fusion," Microelectronics Reliability, vol. 75, pp. 215-222, 2017.

[8] P. C. Tsai, C. C. Cheng, and Y. C. Hwang, "Ball screw preload loss detection using ball pass frequency," Mechanical Systems and Signal Processing, vol. 48, no. 1-2, pp. 77-91, 2014.

[9] G.-H. Feng and Y.-L. Pan, "Establishing a cost-effective sensing system and signal processing method to diagnose preload levels of ball screws," Mechanical Systems and Signal Processing, vol. 28, pp. 78-88, 2012.

[10] C.-F. Han, H.-Q. He, C.-C. Wei et al., "Techniques developed for fault diagnosis of long-range running ball screw drive machine to evaluate lubrication condition," Measurement, vol. 126, pp. 274-288, 2018.

[11] P. Li, X. Jia, J. Feng et al., "Prognosability study of ball screw degradation using systematic methodology," Mechanical Systems and Signal Processing, vol. 109, pp. 45-57, 2018.

[12] Q. Wang and M. Lin, "Electromechanical coupling measurement of a new giant magnetostrictive structure for double-nut ball screw pre-tightening," Measurement Science and Technology, vol. 27, no. 12, article 125906, 2016.

[13] H. T. Feng, Y. L. Wang, C. M. Li, and W. J. Tao, "An automatic measuring method and system using a light curtain for the thread profile of a ballscrew," Measurement Science and Technology, vol. 22, no. 8, article 085106, 2011.

[14] L. Cui, J. Huang, and F. Zhang, "Quantitative and localization diagnosis of a defective ball bearing based on vertical-horizontal synchronization signal analysis," IEEE Transactions on Industrial Electronics, vol. 64, no. 11, pp. 8695-8706, 2017.

[15] L. Guo, Y. Lei, S. Xin, T. Yan, and N. Li, "Deep convolutional transfer learning network: a new method for intelligent fault diagnosis of machines with unlabeled data," IEEE Transactions on Industrial Electronics, vol. 66, no. 9, pp. 7316-7325, 2018.

[16] M. Gupta, J. Gao, C. C. Aggarwal, and J. Han, "Outlier detection for temporal data: a survey," IEEE Transactions on Knowledge and Data Engineering, vol. 26, no. 9, pp. 22502267, 2014.

[17] L. Guo, Y. Lei, N. Li, T. Yan, and N. Li, "Machinery health indicator construction based on convolutional neural networks considering trend burr," Neurocomputing, vol. 292, pp. 142-150, 2018.

[18] Y. Wang, Y. Peng, Y. Zi, X. Jin, and K.-L. Tsui, “A two-stage data-driven-based prognostic approach for bearing degradation 
problem," IEEE Transactions on Industrial Informatics, vol. 12, no. 3, pp. 924-932, 2016.

[19] F. Bonnardot, M. El Badaoui, R. B. Randall, J. Danière, and F. Guillet, "Use of the acceleration signal of a gearbox in order to perform angular resampling (with limited speed fluctuation)," Mechanical Systems and Signal Processing, vol. 19, no. 4, pp. 766-785, 2005.

[20] F. Combet and L. Gelman, "An automated methodology for performing time synchronous averaging of a gearbox signal without speed sensor," Mechanical Systems and Signal Processing, vol. 21, no. 6, pp. 2590-2606, 2007.

[21] F. Combet and R. Zimroz, "A new method for the estimation of the instantaneous speed relative fluctuation in a vibration signal based on the short time scale transform," Mechanical Systems and Signal Processing, vol. 23, no. 4, pp. 1382-1397, 2009.

[22] J. Urbanek, T. Barszcz, and J. Antoni, "A two-step procedure for estimation of instantaneous rotational speed with large fluctuations," Mechanical Systems and Signal Processing, vol. 38, no. 1, pp. 96-102, 2013.

[23] Z. Qiao and Z. Pan, "SVD principle analysis and fault diagnosis for bearings based on the correlation coefficient," Measurement Science and Technology, vol. 26, no. 8, article 085014, 2015.

[24] J. Antoni, "Fast computation of the kurtogram for the detection of transient faults," Mechanical Systems and Signal Processing, vol. 21, no. 1, pp. 108-124, 2007.

[25] L. Stanković, I. Djurović, S. Stanković, M. Simeunović, S. Djukanović, and M. Daković, "Instantaneous frequency in time-frequency analysis: enhanced concepts and performance of estimation algorithms," Digital Signal Processing, vol. 35, pp. 1-13, 2014.

[26] L. Guo, H. Gao, H. Huang, X. He, and S. Li, "Multifeatures fusion and nonlinear dimension reduction for intelligent bearing condition monitoring," Shock and Vibration, vol. 2016, Article ID 4632562, 10 pages, 2016.

[27] V. Climente-Alarcon, J. A. Antonino-Daviu, M. Riera-Guasp, and M. Vlcek, "Induction motor diagnosis by advanced notch FIR filters and the wigner-ville distribution," IEEE Transactions on Industrial Electronics, vol. 61, no. 8, pp. 4217-4227, 2014.

[28] Y. Wang, G. Xu, Q. Zhang, D. Liu, and K. Jiang, "Rotating speed isolation and its application to rolling element bearing fault diagnosis under large speed variation conditions," Journal of Sound and Vibration, vol. 348, pp. 381-396, 2015.

[29] S. Mann and S. Haykin, "The chirplet transform: a generalization of Gabor's logon transform," Vision Interface, vol. 91, pp. 205-212, 1991.

[30] S. Mann and S. Haykin, "Adaptive chirplet: an adaptive generalized wavelet-like transform," Adaptive Signal Processing, vol. 1565, pp. 402-414, 1991.

[31] Y. Yang, Z. K. Peng, G. Meng, and W. M. Zhang, "Characterize highly oscillating frequency modulation using generalized Warblet transform," Mechanical Systems and Signal Processing, vol. 26, pp. 128-140, 2012.

[32] Z. K. Peng, G. Meng, F. L. Chu, Z. Q. Lang, W. M. Zhang, and Y. Yang, "Polynomial chirplet transform with application to instantaneous frequency estimation," IEEE Transactions on Instrumentation and Measurement, vol. 60, no. 9, pp. 32223229, 2011.

[33] Y. Yang, Z. K. Peng, G. Meng, and W. M. Zhang, "Splinekernelled chirplet transform for the analysis of signals with time-varying frequency and its application," IEEE
Transactions on Industrial Electronics, vol. 59, no. 3, pp. 1612-1621, 2012.

[34] S. Mann and S. Haykin, "The chirplet transform: physical considerations," IEEE Transactions on Signal Processing, vol. 43, no. 11, pp. 2745-2761, 1995.

[35] K. I. Rodopoulos and I. A. Antoniadis, "Instantaneous fault frequencies estimation in roller bearings via wavelet structures," Journal of Sound and Vibration, vol. 383, pp. 446-463, 2016. 


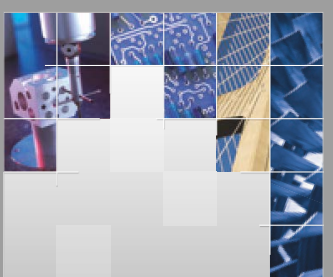

\section{Enfincering}
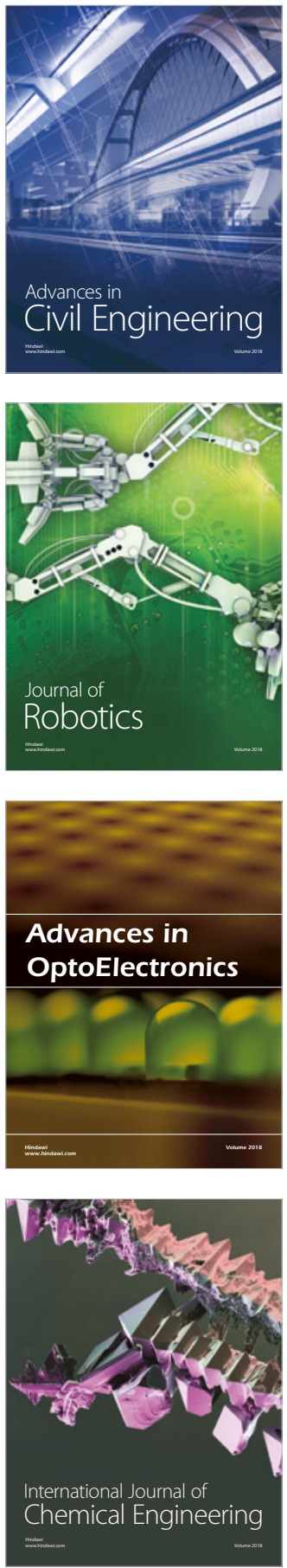

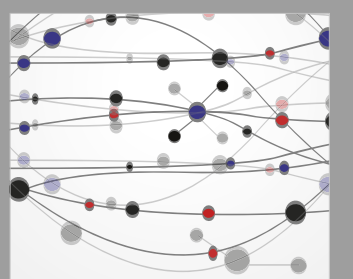

\section{Rotating \\ Machinery}

The Scientific World Journal

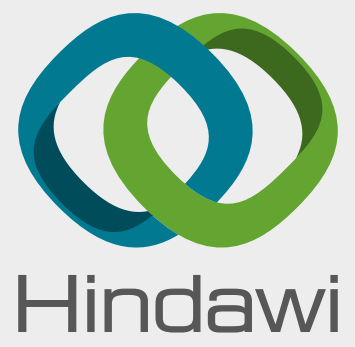

Submit your manuscripts at

www.hindawi.com
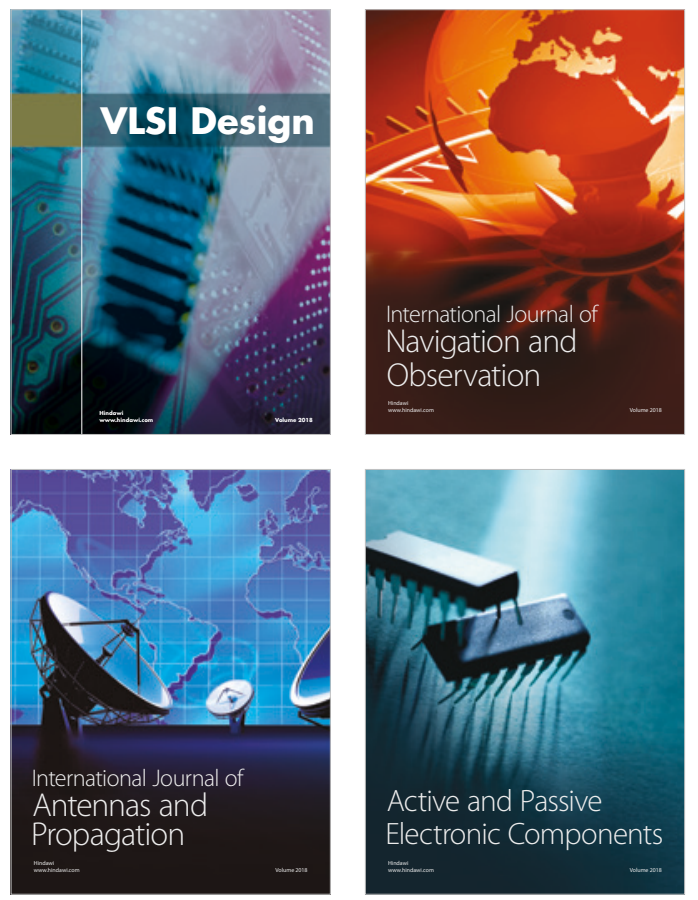
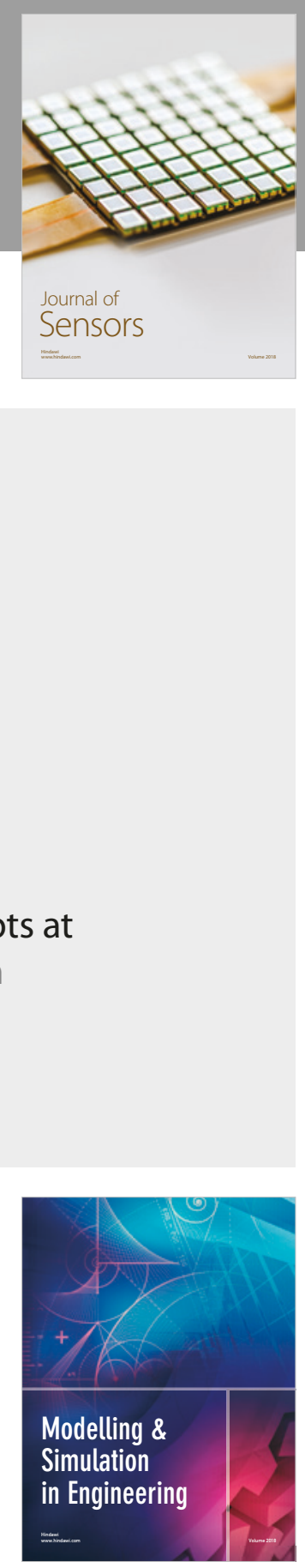

\section{Advances \\ Multimedia}
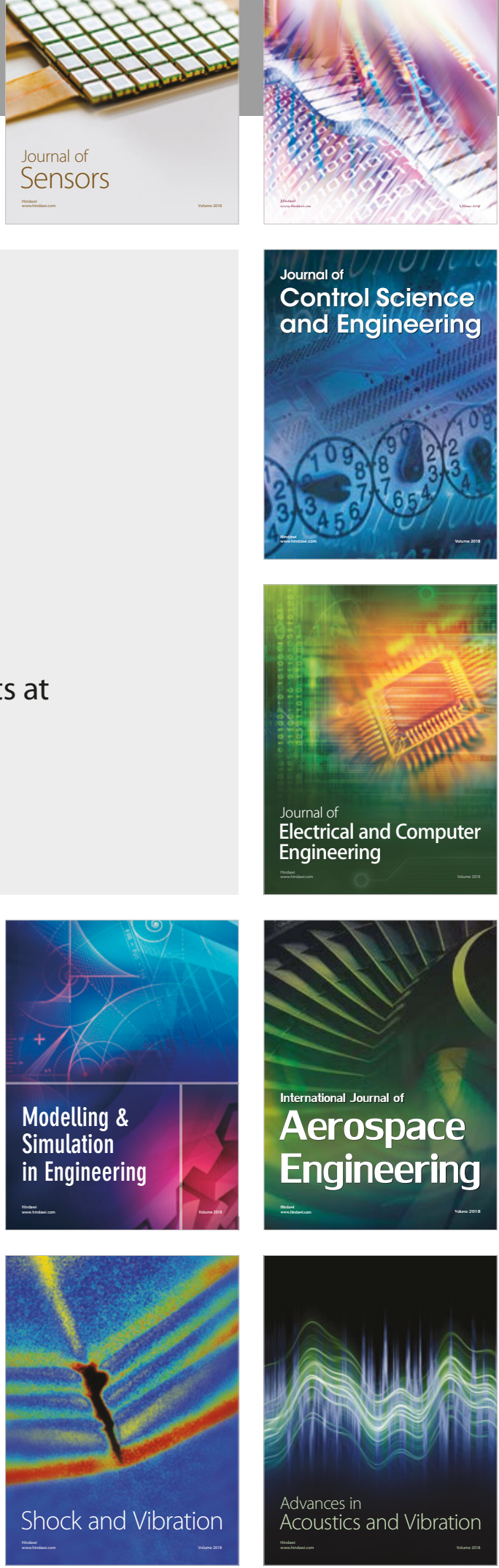